Wick H.,Girard J. and Stahl M.

Prevention of catabolic state during febrile infection in maple sirup urine disease.

In maple sirup urine disease febrile infections endanger life due to the associated catabolic state which raise leucine etc. concentrations to toxic levels. The ratio of glucagon/insulin which is closely related to gluconeogenesis(i.e. protein breakdown) in the catabolic state can be depressed by administration of glucose in sufficient amounts. The catabolic state of fever can be favourably influenced by this simple therapy.
HYPERORNITHINEMIA WITH GYRATE ATROPHY OF THE CHOROID AND RETINA.0.Simell and K. TakKi.Departments of Pediatrics and Ophthalmology, University of Hel. sinki, Helsinki, Finland.

The constant combination of $10-20$ times larger as normal plasma concentration of ornithine with gyrate atrophy of the chorojd and retina was seen in 18 patients, 4 of which were children. The ornithine concentration of urine,spinal fluid and aqueous humour was similarly increased. The height and weight of the patients averaged normal for the Finnish people.Liver and spleen were regularly not enlarged.Hyperammonemia was never found. The fundus of the eye was typically involved containing sharply de ifined areas of choroidal atrophy. Electroretinogram was extinguished and dark adaptation delayed, and the visual fields became extremely narrow with increasing age. All patients were high grade myops.11 out of the 18 had slightly subnormal intelligence.

The metabolic abnormality is suggested to be decreased activity of the enzyme ornithine ketoacid amino. transferase. Autosomal recessive inheritance of the syndrome is obvious.
THE LONG TERM EFFECTS OF A PHENYLALANINE-TYROSINE RESTRICTED DIET IN HEREDITARY TYROSINEMIA. SVerre Halvorsen, Pediatric Research Institute, Rikshospitalet, Oslo, Norway.

Four patients with the chronic form of hereditary tyrosinemia (tyrosinosis) have been followed from 5-10 years. Two of these patients have continuously been on a phetyr restricted diet for 5 and 10 years respectively; the other.two patients were treated with diet for a short time only. The initial response to the diet was marked improvement of the general condition and rapid healing of the tubular dysfunction and the rickets. On long-term diet the general condition remained good and the growth was within normal limits when the diet was successful and no rickets was present. The two patients on a free diet have not thrived as well and the growth has been more retarded. One of them has had severe attacks of abdominal pains and hypertension. No difference has been observed in the iver function in these four patients. With the degree of dietary control obtained in our patients they have thrived wel1; the growth and the tubular function have only slightly been affected, but the liver function has apparently not been influenced by the diet.

TYPE I GLYCOGENOSIS: EFFECTS OF PROLONGED INTRAVENOUS DEXTROSE INFUSION ON GROWTH AND PLASMA BIOCHEMISTRY. A. Macnab. London.

A patient aged 10.7 years with Glycogenos is Type I and having typical abnormalities in plasma biochemistry and severe growth retardation $(16 \mathrm{~cm}$ below third percentilel was treated for 140 days with intravenous dextrose $0.4 \mathrm{G} / \mathrm{kg}$. hr. The infusion was accomplished with a portable infusion pump through an indwelling silastic catheter $(O D=0.5 \mathrm{~mm}$.) The arrangements allowed normal activities and for the child to remain at home. During the infusion an increase in height of $4.8 \mathrm{~cm}$. was observed (1 cm/month) whereas growth in the preceding 12 month $1 \mathrm{~cm}$./month. Within 14 days of starting the infusion values for blood glucose, plasma bicarbonate, lactate pyruvate, cholesterol, uric acid became normal. There was a slower correction of abnormal levels of SGOT and SGPT and incomplete correction of glyceride glycerol and 1 ipoprotein levels. Abnormalities in platelet function were also corrected. Simultaneously the child increased in weight, the liver size decreased, and appetite improved.

\section{METABOLIC PROBLEMS}

SCREENING TESTS FOR THE DETECTION OF HOMOZYGOTES AND HETEROZYGOUS-GENE CARRIERS OF MUCOPOLYSACCHARI DOSES / MPS / BY SO -ISOTOP INCORPORATION METHOD AND SIMPLE STAINING OF ${ }^{4}$ PERIPHERIAL BLOOD SMEAR.

A.Laszlo, A.Mannheim, L.Szabo, Z. Havass, L. Modis, of Szeged/Hungary.

A study was made of 58 heterozygotes and 32 homozygotes of MPS, which had been confirmed by urine analysis and of 20 healthy controls. The method used was a modification of the toluidine blue staining of peripheric blood smears. It was found that this procedure is suitable for the differentiation of MPS hetero- and homozygotes from healthy individuals but not sufficiently specific for differentiation between the individual types or between the heteroand homozygotic character.

In 22 cases s study of the ${ }^{35}$ so incorporation of peripheric white blood cell cultures showed that this method permits differentiation between the hetero-and homozygotes and, further, to a certain extent between the individual types too.
INCREASED EXCRETION OF LOW MOLECULAR WEIGHT GLYCOSAMINOGLYCAN FRAGMENTS FOLLOWING PLASMA INFUSION IN A CASE OF HUNTER SYNDROME. M.F. Dean. P.F. Benson \& Helen Muir, Kennedy Institute of Rheumatology, Bute Gardens, London W6 7DW, U.K. and Paediatric Research Unit, Guy's Hosp. Medical School, London, SEl 9RT, UK A 3-year old boy with the severe form of Hunter syndrome (MPS IIA), was treated by the infusion of plasma from 6 pints of pooled blood over a period of 2 days. Total $24 \mathrm{hr}$ uronic acid excretion increased fourfold immediately following infusion. This increase was almost entirely due to glycosaminoglycan fragments not precipitated with 9-aminoacridine although there was also a small increase in the amount of precipitated (polymeric) material. There was an increase in the proportion of glycosaminoglycan fragments of lowest molecular wt. coupled with a decrease in intermediate sized fragments shown by Sephadex gel filtration. Degree of sulphation was stable before infusion, then fell and rose again in a cyclical pattern after treatment.Larged sized precipitable glycosaminoglycan fragments had a noticably higher content of glucosamine than the smaller supernatant fragments suggesting that the heparan sulphate glycosaminoglycans were less readily degradable than dermatan \& chondroitin sulphates. This pattern of response differed qualitatively from those we have found in 2 types of (MPS III) (Dean et al,1973.) 